\title{
A GENERATOR OF MORPHISMS FOR INFINITE WORDS
}

\author{
PASCAL OCHEM $^{1}$
}

\begin{abstract}
We present an algorithm which produces, in some cases, infinite words avoiding both large fractional repetitions and a given set of finite words. We use this method to show that all the ternary patterns whose avoidability index was left open in Cassaigne's thesis are 2-avoidable. We also prove that there exist exponentially many $\frac{7}{4}^{+}$-free ternary words and $\frac{7}{5}^{+}$-free 4 -ary words. Finally we give small morphisms for binary words containing only the squares $0^{2}, 1^{2}$ and $(01)^{2}$ and for binary words avoiding large squares and fractional repetitions.
\end{abstract}

Mathematics Subject Classification. 68R15.

\section{INTRODUCTION}

We assume that the reader is familiar with Combinatorics on Words (see for instance [11]). A pattern is a finite word of variables. An infinite word $w$ avoids pattern $P$ if for any substitution $\phi$ of the variables of $P$ with non-empty words, $\phi(P)$ is not a factor of $w$. Let $\Sigma_{i}$ denote the $i$-letter alphabet $\{0,1, \ldots, i-1\}$. The avoidability index $\mu(P)$ of $P$ is the smallest integer $k$ such that there exists an infinite word $w$ over $\Sigma_{k}$ avoiding $P$.

In the early 1900's, Thue $[16,17]$ (see also [3]) showed that there exists an infinite word over a three-letter alphabet which contains no square, i.e. two consecutive occurrences of the same factor. He thus proved that $\mu(A A)=3$. He also obtained that $\mu(A A A)=2$.

In 1979, Zimin [18] and Bean, Ehrenfeucht, and McNulty [2] indepently considered the avoidability of patterns and gave a caracterization of the patterns $P$ such that $\mu(P)=\infty$ (unavoidable patterns). For more informations on pattern avoidability, we refer to chapter 3 in [11].

${ }^{1}$ LaBRI, Université Bordeaux I, 351, cours de la Libération, 33405 Talence Cedex, France; ochem@labri.fr

(c) EDP Sciences 2006 
Baker, McNulty, and Taylor [1] found a pattern $P_{4}$ such that $\mu\left(P_{4}\right)=4$ and Clark [5] found a pattern $P_{5}$ such that $\mu\left(P_{5}\right)=5$. The question whether there exists a pattern $P_{k}$ such that $k \leq \mu\left(P_{k}\right)<\infty$ for every $k$ remains open.

Let $t(n)$ be the number of words of length $n$ satisfying a property $\mathcal{P}$. We say that there exist polynomially (resp. exponentially) many words satisfying $\mathcal{P}$ if there exists a constant $c>1$ such that $t(n) \leq n^{c}+c\left(\operatorname{resp} . t(n) \geq c^{n}\right)$ for every $n$. A surprising result in [1] states that there exist polynomially many words avoiding $P_{4}$ over $\Sigma_{4}$.

Cassaigne [4] obtained the avoidability index of every binary pattern and of some ternary patterns. In Section 3, we end the determination of the avoidability index of ternary patterns.

Let $\alpha>1$ be a rational number and let $\ell \geq 1$ be an integer. A word $w$ is an $(\alpha, \ell)$-repetition if we can write it as $w=x^{n} x^{\prime}$ where $x^{\prime}$ is a prefix of $x,|x|=\ell$, and $|w|=\alpha|x|$. Let $\beta$ be a real number and let $n \geq 1$ be an integer. A word is $\left(\beta^{+}, n\right)$-free if it contains no $(\alpha, \ell)$-repetition such that $\alpha>\beta$ and $\ell \geq n$. A word is $\beta^{+}$-free if it is $\left(\beta^{+}, 1\right)$-free. The repetition threshold is the smallest real number $R_{k}$ such that there exists an infinite $R_{k}^{+}$-free word over $\Sigma_{k}$. The exact value of the repetition threshold is known for $2 \leq k \leq 11$ : $R_{2}=2[3,16,17], R_{3}=\frac{7}{4}$ [7], $R_{4}=\frac{7}{5}$ [13], and $R_{k}=\frac{k}{k-1}$ for $5 \leq k \leq 11$ [12]. Dejean [7] conjectured that $R_{k}=\frac{k}{k-1}$ for every $k \geq 5$. In Section 4 , we show that there exist exponentially many $\frac{7}{4}^{+}$-free ternary words and $\frac{7}{5}^{+}$-free 4 -ary words. We use this result to prove that for every pattern $P$ considered in Section 3, there exist exponentially many words avoiding $P$ over $\Sigma_{\mu(P)}$.

The length of a square $u^{2}$ is $|u|$. In Section 5, we give a simple construction for four special types of binary words containing squares of bounded length only.

The main results in Sections 3-5 were obtained using the method presented in Section 2. Notice that we also used this method in [9] to obtain upper bounds on some generalized repetition thresholds.

\section{The Method}

A morphism is $q$-uniform if the image of every letter has length $q$. A uniform morphism $h: \Sigma_{s}^{*} \rightarrow \Sigma_{e}^{*}$ is synchronizing if for any $a, b, c \in \Sigma_{s}$ and $v, w \in \Sigma_{e}^{*}$, if $h(a b)=v h(c) w$, then either $v=\varepsilon$ and $a=c$ or $w=\varepsilon$ and $b=c$.

Lemma 2.1. Let $\alpha, \beta \in \mathbb{Q}, 1<\alpha<\beta<2$ and $n \in \mathbb{N}^{*}$. Let $h: \Sigma_{s}^{*} \rightarrow \Sigma_{e}^{*}$ be a synchronizing q-uniform morphism (with $q \geq 1$ ). If $h(w)$ is $\left(\beta^{+}, n\right)$-free for every $\alpha^{+}$-free word $w$ such that $|w|<\max \left(\frac{2 \beta}{\beta-\alpha}, \frac{2(q-1)(2 \beta-1)}{q(\beta-1)}\right)$, then $h(t)$ is $\left(\beta^{+}, n\right)$-free for every (finite or infinite) word $\alpha^{+}$-free word $t$.

Proof. Suppose $w$ is an $\alpha^{+}$-free word such that $h(w)$ is not $\left(\beta^{+}, n\right)$-free and $w$ is of minimum length with this property. Thus $h(w)$ contains a $\beta^{+}$-repetition, that is, a factor uvu such that $\frac{|u v u|}{|u v|}>\beta$. Denote $x=|u|$ and $y=|v|$. Since $\frac{|u v u|}{|u v|}=\frac{2 x+y}{x+y}>\beta$, we have $y<\frac{2-\beta}{\beta-1} x$. If $x \geq 2 q-1$, then each occurrence of 
$u$ contains at least one full $h$-image of a letter. As $h$ is synchronizing, the two occurrences of $u$ in $u v u$ contain the same $h$-images and in the same positions. Let $U$ be the factor of $w$ that contains all letters whose $h$-images are contained in $u$, and let $V$ be the factor of $w$ that contains all letters whose $h$-images intersect $v$. Denoting $X=|U|$ and $Y=|V|$, we have $Y q<y+2 q$ and $X q>x-2 q$, or equivalently $x<(X+2) q$. Since $U V U$ is a factor of $w$ and $w$ is $\alpha^{+}$-free, then $\frac{2 X+Y}{X+Y} \leq \alpha$, which gives $X \leq \frac{\alpha-1}{2-\alpha} Y$. Now we have

$Y q<y+2 q<\frac{2-\beta}{\beta-1} x+2 q<\frac{2-\beta}{\beta-1}(X+2) q+2 q \leq \frac{2-\beta}{\beta-1}\left(\frac{\alpha-1}{2-\alpha} Y+2\right) q+2 q$,

implying that $Y<\frac{2(2-\alpha)}{\beta-\alpha}$. By the minimality of $w$ we get

$$
|w| \leq 2+Y+2 X \leq 2+Y\left(1+2 \frac{\alpha-1}{2-\alpha}\right)<2+\frac{2(2-\alpha)}{\beta-\alpha} \frac{\alpha}{2-\alpha}=\frac{2 \beta}{\beta-\alpha} .
$$

If $x \leq 2 q-2$, then $y<\frac{2-\beta}{\beta-1}(2 q-2)$ and thus $2 x+y<\frac{2 \beta}{\beta-1}(q-1)$. The minimality of $w$ implies that $(|w|-2) q \leq|u v u|-2=2 x+y-2$. By the above we get that $|w|<\frac{2(q-1)(2 \beta-1)}{q(\beta-1)}$, which completes the proof.

To obtain the results in Sections 3, 4, and 5 we need to construct infinite words over $\Sigma_{e}$ that satisfies a property $\mathcal{P}$ consisting of some $\left(\beta^{+}, n\right)$-freeness properties and the avoidance of a set $S$ of finite words. Such an infinite word is obtained as the $h$-image of any infinite $\alpha^{+}$-free word $t \in \Sigma_{s}^{*}$ by a synchronizing morphism $h: \Sigma_{s}^{*} \rightarrow \Sigma_{e}^{*}$ such that for every $\alpha^{+}$-free word $t \in \Sigma_{s}^{*}, h(t)$ satisfies a property $\mathcal{P}$, where $\mathcal{P}$ consists of some $\left(\beta^{+}, n\right)$-freeness properties and the avoidance of a set $S$ of finite words. It is easy to check that $h$ is synchronizing and that $h(t)$ avoids the set $S$. Using Lemma 2.1 with $\alpha \geq R_{s}$ allows us to check that the $h$-image of any (infinite) $\alpha^{+}$-free word over $\Sigma_{s}$ is $\left(\beta^{+}, n\right)$-free with a finite amount of computation.

We fix $s, q \in \mathbb{N}$ and $\alpha \in \mathbb{Q}$ such that $s \leq 11$ and $R_{s} \leq \alpha<2$, to ensure that there exist an infinite $\alpha^{+}$-free word over $\Sigma_{s}$. We use depth-first search to find a word $w$ over $\Sigma_{e}$ of size $s \times q$ which defines the $q$-uniform morphism $h$ by posing $w=h(0) h(1) \ldots h(s-1)$. Obviously, we can restrict the search to words satisfying $h(s-1) \prec \ldots \prec h(1) \prec h(0)$, where $\prec$ is the lexicographic order of $\Sigma_{e}^{q}$. We prune the search tree by checking property $\mathcal{P}$ on the prefixes of a potential $w$. If no morphism is found, we increase the value of $q$ and try again ${ }^{1}$.

\section{Pattern AVOidance}

We consider here the ternary patterns whose 2-avoidability was left open in Cassaigne's thesis [4]. One of them, namely $A B C B A B C$, is shown to be 2avoidable in [9]. We add to that list the binary pattern $A A B B A$ (resp. $A B A A B$ )

\footnotetext{
${ }^{1}$ The $\mathrm{C}++$ sources of the programs used to find and check the morphisms discussed in this paper are available at: http://dept-info.labri.fr/nochem/morphisms/
} 
which was already known to be 2-avoidable, and is here 2-avoided together with its reverse $A B B A A$ (resp. $A B B A B$ ). In particular, the 2-avoidability of $A B C A C B$ was one of Currie's open problems [6], which was mentioned mainly because $A B C A C B$ and its reverse are not simultaneously 2-avoidable.

Lemma 3.1. Any factor uvu of a $\left(\beta^{+}, n\right)$-free word $w$, with $\beta<2$, is such that

$$
|u| \leq \max \left(n-1-|v|,\left\lfloor\frac{\beta-1}{2-\beta}|v|\right\rfloor\right) .
$$

Proof. If $|u v|<n$ then $|u| \leq n-1-|v|$ and we are done, so suppose $|u v| \geq n$. Since $w$ is $\left(\beta^{+}, n\right)$-free, we have $\frac{|u v u|}{|u v|} \leq \beta \Longrightarrow|u| \leq \frac{\beta-1}{2-\beta}|v|$.

Theorem 3.2. The ternary patterns listed in table 1 are 2-avoidable.

Proof. Suppose that we are given a synchronizing morphism $h: \Sigma_{s}^{*} \rightarrow \Sigma_{e}^{*}$ and a pattern $P$ over the alphabet $\{A, B, \ldots\}$. We can try to prove that $h(t)$ avoids $P$ for every $\alpha^{+}$-free word $t \in \Sigma_{s}^{*}$ in three steps:

(1) Check useful $\left(\beta^{+}, n\right)$-freeness properties of $h(t)$ thanks to Lemma 2.1.

(2) Consider a potential occurrence $\phi(P)$ of $P$, where $\phi$ is a non-erasing morphism. Then use Lemma 3.1 and the results of step (1) to obtain upper bounds on the quantities $a=|\phi(A)|, b=|\phi(B)|, \ldots$

(3) Use the bounds of step (2) to exhaustively check by computer that no occurrence of $P$ appears in $h(t)$.

Let $P^{R}$ denote the reverse of the pattern $P$. We have $\mu\left(P^{R}\right)=\mu(P)$ since a word $w$ avoids $P$ if and only the reverse of $w$ avoids $P^{R}$. Notice that the bounds obtained in step (2) using Lemma 3.1 that hold for potential occurrences of a pattern $P$ also hold for those of $P^{R}$. Thus we try, when possible, to avoid simultaneously $P$ and $P^{R}$. The method discussed in Section 2 is thus used so that the set $S$ contains the small occurrences of $P$ (and maybe $P^{R}$ ). Each line of Table 1 contains one of these pattern $P$ and informations about the morphism $h$ we used to show that $\mu(P)=2$ : the $q$-uniform morphism $h: \Sigma_{s}^{*} \rightarrow \Sigma_{2}^{*}$ is such that for every $\alpha^{+}$-free word $t \in \Sigma_{s}^{*}, h(t)$ avoids $P$. We also precise whether such a word $h(t)$ also avoids $P^{R}$. Now, for each pattern, we give the bounds obtained in step (2) of the proof and the morphism found with the method in Section 2.

The following 8-uniform morphism $h$ is such that for any $\frac{7}{4}^{+}$-free word $t \in \Sigma_{3}^{*}$, $h(t) \in \Sigma_{2}^{*}$ avoids the pattern $A A B A A C B A A B$.

The word $h(t)$ is $\left(\frac{24}{13}^{+}, 3\right)$-free. It contains no square of length at least three, and since the square $A A$ occurs in the pattern, we have that $a \leq 2$. By Lemma 3.1, the factor $B A A B$ implies $b \leq 11 a$. For each occurrence of $B A A B$ appearing in $h(t)$, we have checked that the corresponding occurrence of $A A B A A$ does not appear. For instance, the occurrence $\phi(B A A B)=0110$ (where $\phi(A)=1, \phi(B)=0$ ) 


\begin{tabular}{|l|r|r|r|l|}
\hline Pattern & $s$ & $\alpha$ & $q$ & Comments \\
\hline AABAACBAAB & 3 & $7 / 4$ & 8 & self-reverse \\
\hline AABACCB & 3 & $7 / 4$ & 24 & avoided with its reverse \\
\hline AABBA & 3 & $7 / 4$ & 21 & avoided with its reverse \\
\hline AABBCABBA & 3 & $7 / 4$ & 102 & unavoidable with its reverse \\
\hline AABBCAC & 3 & $7 / 4$ & 86 & avoided with its reverse \\
\hline AABBCBC & 3 & $7 / 4$ & 34 & avoided with its reverse \\
\hline AABBCC & 3 & $7 / 4$ & 52 & self-reverse \\
\hline AABCBC & 3 & $7 / 4$ & 46 & avoided with its reverse \\
\hline AABCCAB & 3 & $7 / 4$ & 34 & avoided with its reverse \\
\hline AABCCBA & 3 & $7 / 4$ & 56 & avoided with its reverse \\
\hline ABAAB & 3 & $7 / 4$ & 10 & avoided with its reverse \\
\hline ABAACBC & 4 & $7 / 5$ & 17 & avoided with its reverse \\
\hline ABAACCB & 3 & $7 / 4$ & 74 & avoided with its reverse \\
\hline ABACACB & 3 & $7 / 4$ & 12 & avoided with its reverse \\
\hline ABACBC & 4 & $7 / 5$ & 29 & self-reverse \\
\hline ABACCAB & 3 & $7 / 4$ & 19 & avoided with its reverse \\
\hline ABACCBA & 3 & $7 / 4$ & 14 & avoided with its reverse \\
\hline ABBACCA & 3 & $7 / 4$ & 12 & self-reverse \\
\hline ABBACCB & 3 & $7 / 4$ & 42 & avoided with its reverse \\
\hline ABBCACB & 4 & $7 / 5$ & 16 & avoided with its reverse \\
\hline ABBCBAC & 4 & $7 / 5$ & 14 & avoided with its reverse \\
\hline ABBCBCA & 3 & $7 / 4$ & 22 & avoided with its reverse \\
\hline ABBCCCAB & 3 & $7 / 4$ & 20 & avoided with its reverse \\
\hline ABCAACB & 3 & $7 / 4$ & 24 & avoided with its reverse \\
\hline ABCACAB & 3 & $7 / 4$ & 10 & avoided with its reverse \\
\hline ABCACB & 6 & $5 / 4$ & 810 & unavoidable with its reverse \\
\hline ABCBBAC & 4 & $7 / 5$ & 18 & avoided with its reverse \\
\hline
\end{tabular}

TABLE 1. Table of 2-avoidable ternary patterns.

appears in $h(t)$, but the factor $\phi(A A B A A)=11011$ does not.

$$
\begin{aligned}
0 & \mapsto 01101011 \\
1 & \mapsto 00111010 \\
2 & \mapsto 00101110
\end{aligned}
$$

The following 24-uniform morphism $h$ is such that for any $\frac{7}{4}^{+}$-free word $t \in \Sigma_{3}^{*}$, $h(t) \in \Sigma_{2}^{*}$ avoids the pattern $A A B A C C B$ and its reverse.

The word $h(t)$ is $\left(\frac{31}{16}^{+}, 3\right)$-free, so $a \leq 2$ and $c \leq 2$. The factor $B A C C B$ implies $b \leq 15(a+2 c)$.

$$
\begin{aligned}
0 & \mapsto 000101001101011001010111 \\
1 & \mapsto 000101000111010111001011 \\
2 & \mapsto 000101000110100111001011
\end{aligned}
$$


The following 21-uniform morphism $h$ is such that for any $\frac{7}{4}^{+}$-free word $t \in \Sigma_{3}^{*}$, $h(t) \in \Sigma_{2}^{*}$ avoids the pattern $A A B B A$ and its reverse.

The word $h(t)$ is $\left(\frac{33}{17}^{+}, 4\right)$-free, so $a \leq 3$ and $b \leq 3$.

$$
\begin{aligned}
0 & \mapsto 001001011100011101101 \\
1 & \mapsto 001000111000101101101 \\
2 & \mapsto 000111000100101101101
\end{aligned}
$$

The following 102-uniform morphism $h$ is such that for any $\frac{7}{4}^{+}$-free word $t \in \Sigma_{3}^{*}$, $h(t) \in \Sigma_{2}^{*}$ avoids the pattern $A A B B C A B B A$.

The word $h(t)$ is $\left(\frac{31}{16}^{+}, 27\right)$-free, so $a \leq 26$ and $b \leq 26$. For each occurrence of $A A B B$ we have checked that the corresponding occurrence of $A B B A$ does not appear. Notice that the $k$-avoidability of $A A B B C A B B A$ implies the $k$-avoidability of $A A B B A$. A simple backtracting algorithm shows that $A A B B C A B B A$ and $A B B A A$ (i.e. the reverse of $A A B B A$ ) are not simultaneously 2-avoidable, so that the two previous results are tight, in a way.

$$
\begin{gathered}
0 \mapsto 0001000101101110111000101100010001011011101100010110111000101101 \\
11011100010110111011000101101110001011 \\
1 \mapsto 0001000101101110110001011011101110001011000100010110111000101101 \\
11011100010110111011000101101110001011 \\
2 \mapsto 0001000101101110001011011101110001011000100010110111011000101101 \\
11011100010110111011000101101110001011
\end{gathered}
$$

The following 86-uniform morphism $h$ is such that for any $\frac{7}{4}^{+}$-free word $t \in \Sigma_{3}^{*}$, $h(t) \in \Sigma_{2}^{*}$ avoids the pattern $A A B B C A C$ and its reverse.

The word $h(t)$ is $\left(\frac{43}{22}^{+}, 3\right)$-free, so $a \leq 2$ and $b \leq 2$.

The factor $C A C$ implies $c \leq \max (2-a, 21 a)=21 a$.

$$
\begin{gathered}
0 \mapsto 00010101100101001101010110010101001101011001010011010 \\
100010111010100110101100101011101 \\
1 \mapsto 00010101100101001101010001011101010011010110010101001 \\
101010110010100110101100101011101 \\
2 \mapsto 00010101100101001101010001011101010011010110010100110 \\
101011001010100110101100101011101
\end{gathered}
$$

The following 34-uniform morphism $h$ is such that for any $\frac{7}{4}^{+}$-free word $t \in \Sigma_{3}^{*}$, $h(t) \in \Sigma_{2}^{*}$ avoids the pattern $A A B B C B C$ and its reverse.

The word $h(t)$ is $\left(\frac{33}{17}^{+}, 3\right)$-free, so $a \leq 2$ and $b=c=1$.

$$
\begin{aligned}
& 0 \mapsto 1110101110001010001110001010100011 \\
& 1 \mapsto 1110101110001010100011100010100011 \\
& 2 \mapsto 1110101110001110101000111000101000
\end{aligned}
$$


The following 52-uniform morphism $h$ is such that for any $\frac{7}{4}^{+}$-free word $t \in \Sigma_{3}^{*}$, $h(t) \in \Sigma_{2}^{*}$ avoids the pattern $A A B B C C$.

The word $h(t)$ is $\left(\frac{59}{30}^{+}, 3\right)$-free, so $a \leq 2, b \leq 2$, and $c \leq 2$.

$$
\begin{aligned}
& 0 \mapsto 1101001110001101000101100011010011100101100011100101 \\
& 1 \mapsto 1101001110001101000101100011100101100011010011100101 \\
& 2 \mapsto 1101001110001101000101100011100101110100111001011000
\end{aligned}
$$

The following 46-uniform morphism $h$ is such that for any $\frac{7}{4}^{+}$-free word $t \in \Sigma_{3}^{*}$, $h(t) \in \Sigma_{2}^{*}$ avoids the pattern $A A B C B C$ and its reverse.

The word $h(t)$ is $\left(\frac{367}{184}^{+}, 3\right)$-free, so $a \leq 2$ and $b=c=1$. Notice that the only occurrences of $A A B B$ in $h(t)$ are 0011 and 1100.

$$
\begin{aligned}
& 0 \mapsto 0011010011100011001011000110100110001110010110 \\
& 1 \mapsto 0011010011100011001011000110100111001011000111 \\
& 2 \mapsto 0011010011100011001011000111001101001110010110
\end{aligned}
$$

The following 34-uniform morphism $h$ is such that for any $\frac{7}{4}^{+}$-free word $t \in \Sigma_{3}^{*}$, $h(t) \in \Sigma_{2}^{*}$ avoids the pattern $A A B C C A B$ and its reverse.

The word $h(t)$ is $\left(\frac{257}{136}^{+}, 4\right)$-free, so $a \leq 3$ and $c \leq 3$. The factor $A B C C A B$ implies $a+b \leq\left\lfloor\frac{242}{15} c\right\rfloor$, thus $b \leq\left\lfloor\frac{242}{15} c\right\rfloor-a$.

$$
\begin{aligned}
& 0 \mapsto 0000101111101000011111101000101111 \\
& 1 \mapsto 0000101110100001111010001011111100 \\
& 2 \mapsto 0000101110100000011110100010111111
\end{aligned}
$$

The following 56-uniform morphism $h$ is such that for any $\frac{7}{4}^{+}$-free word $t \in \Sigma_{3}^{*}$, $h(t) \in \Sigma_{2}^{*}$ avoids the pattern $A A B C C B A$ and its reverse.

The word $h(t)$ is $\left(\frac{36}{19}^{+}, 3\right)$-free, so $a \leq 2$ and $c \leq 2$. The factor $B C C B$ implies $b \leq 17 c$.

$$
\begin{aligned}
& 0 \mapsto 00010110001110010111010011100011010001011000111001011101 \\
& 1 \mapsto 00010110001101001110010110001101000101110100111001011101 \\
& 2 \mapsto 00010110001101000101110100111001011000110100111001011101
\end{aligned}
$$

The following 10-uniform morphism $h$ is such that for any $\frac{7}{4}^{+}$-free word $t \in \Sigma_{3}^{*}$, $h(t) \in \Sigma_{2}^{*}$ avoids the pattern $A B A A B$ and its reverse.

The word $h(t)$ is $\left(\frac{39}{20}^{+}, 3\right)$-free, so $a \leq 2$. The factor $B A A B$ implies $b \leq 38 a$.

$$
\begin{aligned}
& 0 \mapsto 0001110101 \\
& 1 \mapsto 0000111101 \\
& 2 \mapsto 0000101111
\end{aligned}
$$


The following 17-uniform morphism $h$ is such that for any $\frac{7}{5}^{+}$-free word $t \in \Sigma_{4}^{*}$, $h(t) \in \Sigma_{2}^{*}$ avoids the pattern $A B A A C B C$ and its reverse.

The word $h(t)$ is $\left(\frac{13}{7}^{+}, 7\right)$-free, so $a \leq 6$. The word $h(t)$ is $\left(\frac{23}{16}^{+}, 20\right)$-free. Suppose $b+c \geq 20$, then the factor $C B C$ implies $c \leq\left\lfloor\frac{7}{9} b\right\rfloor$ and the factor $B A A C B$ implies $b \leq\left\lfloor\frac{7}{9}(2 a+c)\right\rfloor$. From these relations we can deduce $b \leq 22$ and $c \leq 17$. Thus we have $b \leq 22$ and $c \leq 18$.

$$
\begin{aligned}
& 0 \mapsto 01110000110000111 \\
& 1 \mapsto 01011100110011101 \\
& 2 \mapsto 01000110011000101 \\
& 3 \mapsto 00011110011110001
\end{aligned}
$$

The following 74-uniform morphism $h$ is such that for any $\frac{7}{4}^{+}$-free word $t \in \Sigma_{3}^{*}$, $h(t) \in \Sigma_{2}^{*}$ avoids the pattern $A B A A C C B$ and its reverse.

The word $h(t)$ is $\left(\frac{193}{104}^{+}, 3\right)$-free, so $a \leq 2$ and $c \leq 2$. The factor $B A A C C B$ implies $b \leq\left\lfloor\frac{89}{15}(a+c)\right\rfloor$.

$$
\begin{gathered}
0 \mapsto 000001011001110100110101100101110011010000011111010011000 \\
10100011001011111 \\
1 \mapsto 000001011001010100110001011001010011010000011111010011000 \\
10100011001011111 \\
2 \mapsto 000001011001010011010001100101010011010000011111010011000 \\
10100011001011111
\end{gathered}
$$

The following 12-uniform morphism $h$ is such that for any $\frac{7}{4}^{+}$-free word $t \in \Sigma_{3}^{*}$, $h(t) \in \Sigma_{2}^{*}$ avoids the pattern $A B A C A C B$ and its reverse.

The word $h(t)$ is $\left(\frac{15}{8}^{+}, 3\right)$-free, so $a=c=1$. The factor $B A C A C B$ implies $b \leq 14(a+c)=28$.

$$
0 \mapsto 001010011111 \quad 1 \mapsto 000110100111 \quad 2 \mapsto 000001101011
$$

The following 29-uniform morphism $h$ is such that for any $\frac{7}{5}^{+}$-free word $t \in \Sigma_{4}^{*}$, $h(t) \in \Sigma_{2}^{*}$ avoids the pattern $A B A C B C$.

The word $h(t)$ is $\left(\frac{41}{29}^{+}, 291\right)$-free. Suppose $a+b \geq 291$ and $b+c \geq 291$. The factors $A B A, C B C$, and $B A C B$ respectively imply that $17 a \leq 12 b(i), 17 c \leq 12 b(i i)$, and $17 b \leq 12(a+c)$ ( iii $)$. The combination $17 \times(i)+17 \times(i i)+24 \times($ iii $)$ gives $a+c \leq 0$, a contradiction. So we can suppose without loss of generality that $b+c \leq 290(i v)$. If $291 \leq a+b(v)$ then $(i)$ and $(i i i)$ still hold and the combination $2324 \times(i)+1649 \times(i i i)+19788 \times(i v)+19720 \times(v)$ gives $213 b \leq 0$. 
This contradiction shows that $a+b \leq 290$ and $b+c \leq 290$.

$$
\begin{aligned}
& 0 \mapsto 00011010110000111100101001110 \\
& 1 \mapsto 00011010110000011100101001111 \\
& 2 \mapsto 00001101011000111101110011110 \\
& 3 \mapsto 00001101011000011110011101111
\end{aligned}
$$

The following 19-uniform morphism $h$ is such that for any $\frac{7}{4}^{+}$-free word $t \in \Sigma_{3}^{*}$, $h(t) \in \Sigma_{2}^{*}$ avoids the pattern $A B A C C A B$ and its reverse.

The word $h(t)$ is $\left(\frac{35}{19}^{+}, 5\right)$-free, so $c \leq 4$. The factor $A C C A$ implies $a \leq \max (4-2 c$, $\left.\left\lfloor\frac{32}{3} c\right\rfloor\right)=\left\lfloor\frac{32}{3} c\right\rfloor$. The factor $A B A C C A B$ implies $a+b \leq\left\lfloor\frac{16}{3}(a+2 c)\right\rfloor$, thus $b \leq$ $\left\lfloor\frac{13 a+32 c}{3}\right\rfloor$.

$$
\begin{aligned}
0 & \mapsto 0101110010100000111 \\
1 & \mapsto 0101100000010011100 \\
2 & \mapsto 0100010111010100011
\end{aligned}
$$

The following 14-uniform morphism $h$ is such that for any $\frac{7}{4}^{+}$-free word $t \in \Sigma_{3}^{*}$, $h(t) \in \Sigma_{2}^{*}$ avoids the pattern $A B A C C B A$ and its reverse.

If $w$ is an occurrence of $A B A C C B A$ such that $a>1$, then the suffix of $w$ of size $|w|-a+1$ is a smaller occurrence of $A B A C C B A$ such that $a=1$. So we assume without loss of generality that $a=1$. The word $h(t)$ is $\left(\frac{23}{12}^{+}, 3\right)$-free, so $c \leq 2$. The factor $B A C C B A$ implies $a+b \leq 22 c$, thus $b \leq 22 c-1$.

$$
\begin{aligned}
& 0 \mapsto 10101100001110 \\
& 1 \mapsto 01010111100011 \\
& 2 \mapsto 01010000111110
\end{aligned}
$$

The following 12-uniform morphism $h$ is such that for any $\frac{7}{4}^{+}$-free word $t \in \Sigma_{3}^{*}$, $h(t) \in \Sigma_{2}^{*}$ avoids the pattern $A B B A C C A$.

The word $h(t)$ is $\left(\frac{31}{16}^{+}, 4\right)$-free, so $b \leq 3$ and $c \leq 3$. The factor $A B B A$ implies $a \leq \max (3-2 b, 30 b)=30 b$.

$$
\begin{aligned}
0 & \mapsto 000111001011 \\
1 & \mapsto 000101111010 \\
2 & \mapsto 000100111011
\end{aligned}
$$

The following 42-uniform morphism $h$ is such that for any $\frac{7}{4}^{+}$-free word $t \in \Sigma_{3}^{*}$, $h(t) \in \Sigma_{2}^{*}$ avoids the pattern $A B B A C C B$ and its reverse.

The word $h(t)$ is $\left(\frac{53}{28}^{+}, 3\right)$-free, so $b \leq 2$ and $c \leq 2$. The factor $A B B A$ implies $a \leq\left\lfloor\frac{50}{3} b\right\rfloor$.

$$
\begin{aligned}
& 0 \mapsto 000010111100010111010001111010000111010111 \\
& 1 \mapsto 000010111100010101100101001101011001010111 \\
& 2 \mapsto 000010111100010100011110100001110100010111
\end{aligned}
$$


The following 16-uniform morphism $h$ is such that for any $\frac{7}{5}^{+}$-free word $t \in \Sigma_{4}^{*}$, $h(t) \in \Sigma_{2}^{*}$ avoids the pattern $A B B C A C B$ and its reverse.

The word $h(t)$ is $\left(\frac{9}{5}^{+}, 4\right)$-free, so $b \leq 3$. The word $h(t)$ is $\left(\frac{233}{160}^{+}, 49\right)$-free. Suppose $a+c \geq 49$. The factors $A B B C A$ and $C A C$ respectively imply that $a \leq\left\lfloor\frac{73}{87}(2 b+c)\right\rfloor$ and $c \leq\left\lfloor\frac{73}{87} a\right\rfloor$. From these relations we can deduce $a \leq 15$ and $c \leq 12$. This contradiction shows that $a+c \leq 48$.

$$
\begin{aligned}
0 & \mapsto 0010000001101111 \\
1 & \mapsto 0000111010001111 \\
2 & \mapsto 0000100111111011 \\
3 & \mapsto 0000001001101011
\end{aligned}
$$

The following 14-uniform morphism $h$ is such that for any $\frac{7}{5}^{+}$-free word $t \in \Sigma_{4}^{*}$, $h(t) \in \Sigma_{2}^{*}$ avoids the pattern $A B B C B A C$ and its reverse.

The word $h(t)$ is $\left(\frac{18}{11}^{+}, 7\right)$-free, so $b \leq 6$. The word $h(t)$ is $\left(\frac{29}{20}^{+}, 43\right)$-free. Suppose $a+b+c \geq 43$. The factors $A B B C B A$ and $C B A C$ respectively imply that $a \leq$ $\left\lfloor\frac{9}{11}(3 b+c)\right\rfloor$ and $c \leq\left\lfloor\frac{9}{11}(a+b)\right\rfloor$. From these relations we can deduce $a \leq 54$ and $c \leq 49$.

$$
\begin{aligned}
& 0 \mapsto 00101010101011 \\
& 1 \mapsto 00010001110111 \\
& 2 \mapsto 00000101011111 \\
& 3 \mapsto 00000010111111
\end{aligned}
$$

The following 22-uniform morphism $h$ is such that for any $\frac{7}{4}^{+}$-free word $t \in \Sigma_{3}^{*}$, $h(t) \in \Sigma_{2}^{*}$ avoids the pattern $A B B C B C A$ and its reverse.

The word $h(t)$ is $\left(\frac{173}{88}^{+}, 3\right)$-free, so $b=c=1$. The factor $A B B C B C A$ implies $a \leq\left\lfloor\frac{85}{3}(3 b+2 c)\right\rfloor=141$.

$$
\begin{aligned}
0 & \mapsto 0001101011001010100111 \\
1 & \mapsto 0001101010110010100111 \\
2 & \mapsto 0001101010011100101011
\end{aligned}
$$

The following 20-uniform morphism $h$ is such that for any $\frac{7}{4}^{+}$-free word $t \in \Sigma_{3}^{*}$, $h(t) \in \Sigma_{2}^{*}$ avoids the pattern $A B B C C A B$ and its reverse.

The word $h(t)$ is $\left(\frac{15}{8}^{+}, 4\right)$-free, so $b \leq 3$ and $c \leq 3$. The factor $A B B C C A B$ implies $a+b \leq 7(b+2 c)$, thus $a \leq 6 b+14 c$.

$$
\begin{aligned}
& 0 \mapsto 00010100100101011111 \\
& 1 \mapsto 00010010001110110111 \\
& 2 \mapsto 00000101011011010111
\end{aligned}
$$

The following 24-uniform morphism $h$ is such that for any $\frac{7}{4}^{+}$-free word $t \in \Sigma_{3}^{*}$, $h(t) \in \Sigma_{2}^{*}$ avoids the pattern $A B C A A C B$ and its reverse. 
The word $h(t)$ is $\left(\frac{187}{96}^{+}, 4\right)$-free, so $a \leq 3$. The word $h(t)$ is $\left(\frac{355}{192}^{+}, 97\right)$-free. The factor $C A A C$ implies $c \leq \max \left(96-2 a,\left\lfloor\frac{326 a}{29}\right\rfloor\right)=96-2 a$. The factor $B C A A C B$ implies $b \leq \max \left(96-2 a-2 c,\left\lfloor\frac{326(a+c)}{29}\right\rfloor\right)$.

$$
\begin{aligned}
& 0 \mapsto 000001011111001000110111 \\
& 1 \mapsto 000001011111000100111011 \\
& 2 \mapsto 000001010111110010011011
\end{aligned}
$$

The following 10-uniform morphism $h$ is such that for any $\frac{7}{4}^{+}$-free word $t \in \Sigma_{3}^{*}$, $h(t) \in \Sigma_{2}^{*}$ avoids the pattern $A B C A C A B$ and its reverse.

The word $h(t)$ is $\left(\frac{79}{40}^{+}, 3\right)$-free, so $a=c=1$. The word $h(t)$ is $\left(\frac{149}{80}^{+}, 41\right)$ free. The factor $A B C A C A B$ implies $a+b \leq \max \left(40-a-2 c,\left\lfloor\frac{69}{11}(a+2 c)\right\rfloor\right)$, thus $b \leq 40-2 a-2 c=36$.

$$
0 \mapsto 0001110101 \quad 1 \mapsto 0001011101 \quad 2 \mapsto 0001010111
$$

The 810-uniform morphism $h=m_{4,2} \circ m_{6,4}$ is such that for any $\frac{5}{4}^{+}$-free word $t \in \Sigma_{6}^{*}, h(t) \in \Sigma_{2}^{*}$ avoids the pattern $A B C A C B$.

The word $h(t)$ is $\left(\frac{1073}{810}^{+}, 3241\right)$-free. Suppose $a+c \geq 3241$. The factors $A B C A$, $B C A C B$, and $C A C$ respectively imply that $547 a \leq 263(b+c)(i), 547 b \leq 263(a+$ 2c) (ii), and $547 c \leq 263 a$ (iii). The combination $2 \times(i)+($ ii $)+2 \times($ iii $)$ gives $305 a+568 b+42 c \leq 0$. This contradiction shows that $a+c \leq 3240$ (iv). The word $h(t)$ is $\left(\frac{29}{14}^{+}, 4\right)$-free, so the factor $C A C$ implies $c \leq 14 a(v)$. Suppose now $3238 \leq b(v i)$, so that $a+b+2 c \geq 3241$ and (ii) still holds. The combination $15 \times(i i)+7627 \times(i v)+263 \times(v)+7632 \times(v i)$ gives $573 b+936 \leq 0$. This contradiction shows that $b \leq 3237$.

The 135-uniform morphism $m_{4,2}$ is given by:

$0 \mapsto 00000100111101100010111100000101101000011111011000010011110000010$ 1101000011111010010111100001001111101100000100111010000111110100101111 $1 \mapsto 00000100111101100010111100000101101000011111011000010011110000010$ 1101000011110110000010011111011000101111000001011010000111110100101111 $2 \mapsto 00000100111101100001111101001011110000100111110110000010011101000$ 0111110100101111000001011010000111110110000100111010000111110100101111 $3 \mapsto 00000100111101100001111101001011110000010110100001111011000001001$ 1111011000101111000001011010000111110110000100111010000111110100101111

The 6-uniform morphism $m_{6,4}$ is given by:

$$
\begin{array}{lll}
0 \mapsto 032131 & 1 \mapsto 031232 & 2 \mapsto 023121 \\
3 \mapsto 021323 & 4 \mapsto 013212 & 5 \mapsto 012313
\end{array}
$$


The following 18-uniform morphism $h$ is such that for any $\frac{7}{5}^{+}$-free word $t \in \Sigma_{4}^{*}$, $h(t) \in \Sigma_{2}^{*}$ avoids the pattern $A B C B B A C$ and its reverse.

The word $h(t)$ is $\left(\frac{8}{5}^{+}, 7\right)$-free, so $b \leq 6$. The word $h(t)$ is $\left(\frac{527}{378}^{+}, 181\right)$-free. Suppose $a+2 b+c \geq 181$. The factors $C B B A C$ and $A B C B B A$ respectively imply that $c \leq\left\lfloor\frac{149}{229}(a+2 b)\right\rfloor$ and $a \leq\left\lfloor\frac{149}{229}(3 b+c)\right\rfloor$. From these relations we can deduce $a \leq 28$ and $c \leq 26$. This contradiction shows that $a+2 b+c \leq 180$.

$$
\begin{aligned}
& 0 \mapsto 010000011011011011 \\
& 1 \mapsto 001001001001111101 \\
& 2 \mapsto 001000000111111011 \\
& 3 \mapsto 000000101010111111
\end{aligned}
$$

\section{Applichtion to REPETItion-Free WORDS}

The repetition threshold for binary words is 2 , and this result is tight in the following senses:

(1) There exist polynomially many $2^{+}$-free binary words.

(2) There exist arbitrarily large squares in any infinite $2^{+}$-free binary word.

In this section we show that no similar situation occurs for ternary and 4-ary words. We use the following easy lemma, which is already implicitly used in [10].

Lemma 4.1. There are at least $2^{\left\lceil\frac{n}{k}\right\rceil} R_{k}^{+}$-free words of length $n$ over $\Sigma_{k+1}$.

Proof. Consider an $R_{k}^{+}$-free word $w$ in $\Sigma_{k}^{n}$. At least one letter in $\Sigma_{k}$, say 0 , occurs at least $\left\lceil\frac{n}{k}\right\rceil$ times in $w$. The letter $k$ belongs to $\Sigma_{k+1}$ but does not belong to $\Sigma_{k}$. Notice that replacing zero or more occurrences of 0 by an occurrence of $k$ in $w$ produces an $R_{k}^{+}$-free word of length $n$ over $\Sigma_{k+1}$, and that we can obtain at least $2^{\left\lceil\frac{n}{k}\right\rceil}$ such words.

\section{Theorem 4.2.}

(1) There exist exponentially many $\frac{7}{4}^{+}$-free ternary words with no large repetition of exponent $\frac{7}{4}$.

(2) There exist exponentially many $\frac{7}{5}^{+}$-free 4-ary words with no large repetition of exponent $\frac{7}{5}$.

Proof. By Lemma 4.1, there exist exponentially many $\frac{7}{5}^{+}$-free words over $\Sigma_{5}$ and exponentially many $\frac{5}{4}^{+}$-free words over $\Sigma_{6}$. 
The following 59-uniform morphism $h$ is such that for any $\frac{7}{5}^{+}$-free word $t \in \Sigma_{5}^{*}$, $h(t) \in \Sigma_{3}^{*}$ is $\frac{7}{4}^{+}$-free and $\left(\frac{3}{2}^{+}, 10\right)$-free.

$$
\begin{aligned}
& 0 \mapsto 01020121021201210120102101202102012102120210120102101210212 \\
& 1 \mapsto 01020120210201210212012101201021202102012021201210201021012 \\
& 2 \mapsto 01020120210201210120102101202120121021202102012021201021012 \\
& 3 \mapsto 01020120210201021201210201202101201021012102120210201021012 \\
& 4 \mapsto 01020120210121020102120121012021020120212012102010212021012
\end{aligned}
$$

The following 132-uniform morphism $h$ is such that for any $\frac{5}{4}^{+}$-free word $t \in \Sigma_{6}^{*}$, $h(t) \in \Sigma_{4}^{*}$ is $\frac{7}{5}^{+}$-free and $\left(\frac{61}{44}^{+}, 11\right)$-free.

$0 \mapsto 0120310213201230210320130231203213012310213203123013210312013021$
03201231021301203210231201321031230132031021301231032013023120321023
$1 \mapsto 0120310213201230210320130231032130123102132031230132102312013021$
03201230213203102301203210231201321031230213201231032130231201321023
$2 \mapsto 0120310213201230210320130231032130120310230132103120130231032012$
30213203102301203210231201321031230132031021301231032130231201321023
$3 \mapsto 0120310213201230210312013210231203213023103201230213203102301321$
03120130210320123102132031230132102312032130231032013021031201321023
$4 \mapsto 0120310213201230210312013023103213012310213203123013210312013021$
03201230213203102301203213023120132103123021320123103213023120321023
$5 \mapsto 0120310213201230210312013023103213012031023013210312013021032012$
30213203102301203210231201302103123013203102130123103213023120321023

Corollary 4.3. For each pattern $P$ listed in Table 1, there exist exponentially many words avoiding $P$ over $\Sigma_{2}$.

Proof. Binary words avoiding $A B C A C B$ are constructed from $\frac{5}{4}^{+}$-free words over $\Sigma_{5}$, and there are exponentially many such words by Lemma 4.1 . For each other pattern $P$ listed in Table 1, binary words avoiding $P$ are constructed from either $\frac{7}{4}^{+}$-free words over $\Sigma_{3}$, or $\frac{7}{5}^{+}$-free words over $\Sigma_{4}$. In both cases, there are exponentially many such words by Theorem 4.2 .

We have not been able to extend Theorem 4.2 to $\Sigma_{5}$. However, we believe that the following strong form of Dejean's conjecture holds.

Conjecture 4.4. For every $k \geq 5$, there exist exponentially many $\frac{k}{k-1}^{+}$-free words over $\Sigma_{k}$. 


\section{Binary WORDS AVOIDING LARGE SQUARES}

Fraenkel and Simpson constructed in [8] an infinite binary word containing only three squares. Another construction using uniform morphisms is given in [14]. Shallit [15] also gives uniform morphisms for binary words avoiding:

- squares of length at least 3 and $3^{+}$-repetitions (10-uniform);

- squares of length at least 4 and $\frac{5}{2}^{+}$-repetitions (1560-uniform);

- squares of length at least 7 and $\frac{7}{3}^{+}$-repetitions (252-uniform).

In this section we give small $\Sigma_{3}^{*} \rightarrow \Sigma_{2}^{*}$ uniform morphisms producing words having these properties.

The following 50-uniform morphism $h$ is such that for any $\frac{7}{4}^{+}$-free word $t \in \Sigma_{3}^{*}$, $h(t) \in \Sigma_{2}^{*}$ contains only the squares in $\left\{0^{2}, 1^{2},(01)^{2}\right\}$ and is $\left(\frac{37}{19}^{+}, 3\right)$-free.

$$
\begin{aligned}
& 0 \mapsto 00011001011000111001011001110001011100101100010111 \\
& 1 \mapsto 00011001011000101110010110011100010110001110010111 \\
& 2 \mapsto 00011001011000101110010110001110010111000101100111
\end{aligned}
$$

The following 8-uniform morphism $h$ is such that for any $\frac{7}{4}^{+}$-free word $t \in \Sigma_{3}^{*}$, $h(t) \in \Sigma_{2}^{*}$ is $3^{+}$-free, $\left(\frac{5}{2}^{+}, 2\right)$-free, and $\left(\frac{59}{32}^{+}, 3\right)$-free.

$$
0 \mapsto 01101011 \quad 1 \mapsto 00111010 \quad 2 \mapsto 00101110
$$

The following 103-uniform morphism $h$ is such that for any $\frac{7}{4}^{+}$-free word $t \in \Sigma_{3}^{*}$, $h(t) \in \Sigma_{2}^{*}$ is $\frac{5}{2}^{+}$-free, $\left(\frac{7}{3}^{+}, 3\right)$-free, and $\left(\frac{823}{412}^{+}, 4\right)$-free.

$$
\begin{gathered}
0 \mapsto 0010011010010110010011011001010011010110010011011001011010011011 \\
001001101011001010011011001011010011011 \\
1 \mapsto 0010011010010110010011011001010011010110010011011001011010011011 \\
001001101001011001001101011001010011011 \\
2 \mapsto 0010011010010110010011011001010011010110010011010010110010011011 \\
001011010011011001001101011001010011011
\end{gathered}
$$

The following 30-uniform morphism $h$ is such that for any $\frac{7}{4}^{+}$-free word $t \in \Sigma_{3}^{*}$, $h(t) \in \Sigma_{2}^{*}$ is $\frac{7}{3}^{+}$-free and $\left(\frac{79}{40}^{+}, 7\right)$-free.

$$
\begin{aligned}
& 0 \mapsto 001011001011010011011001001101 \\
& 1 \mapsto 001011001011010011001011001101 \\
& 2 \mapsto 001011001001101100101101001101
\end{aligned}
$$




\section{REFERENCES}

[1] K.A. Baker, G.F. McNulty and W. Taylor, Growth Problems for Avoidable Words. Theoret. Comput. Sci. 69 (1989) 319-345.

[2] D.R. Bean, A. Ehrenfeucht, G.F. McNulty, Avoidable Patterns in Strings of Symbols. Pacific J. Math. 85 (1979) 261-294.

[3] J. Berstel, Axel Thue's Papers on Repetitions in Words: a Translation. Number 20 in Publications du Laboratoire de Combinatoire et d'Informatique Mathématique. Université du Québec à Montréal (February 1995).

[4] J. Cassaigne, Motifs évitables et régularité dans les mots, Thèse de Doctorat, Université Paris VI (Juillet 1994).

[5] R.J. Clark. Avoidable formulas in combinatorics on words, Ph.D. Thesis, University of California, Los Angeles (2001).

[6] J.D. Currie, Open problems in pattern avoidance. Amer. Math. Monthly 100 (1993) 790-793.

[7] F. Dejean, Sur un théorème de Thue. J. Combin. Theory. Ser. A 13 (1972) 90-99.

[8] A.S. Fraenkel and R.J. Simpson, How many squares must a binary sequence contain? Elect. J. Combin. 2 (1995) \#R2.

[9] L. Ilie, P. Ochem and J.O. Shallit, A generalization of Repetition Threshold. Theoret. Comput. Sci. 345 (2005) 359-369.

[10] J. Karhumäki and J. O. Shallit, Polynomial versus exponential growth in repetition-free binary words. J. Combin. Theory Ser. A 105 (2004) 335-347.

[11] M. Lothaire, Algebraic Combinatorics on Words. Cambridge Univ. Press (2002).

[12] J. Moulin-Ollagnier, Proof of Dejean's conjecture for alphabets with 5,6,7,8,9,10 and 11 letters. Theoret. Comput. Sci. 95 (1992) 187-205.

[13] J.-J. Pansiot, A propos d'une conjecture de F. Dejean sur les répétitions dans les mots. Disc. Appl. Math. 7 (1984) 297-311.

[14] N. Rampersad, J. Shallit and M.-W. Wang, Avoiding large squares in infinite binary words. Theoret. Comput. Sci. 339 (2005) 19-34.

[15] J.O. Shallit, Simultaneous avoidance of large squares and fractional powers in infinite binary words. Internat. J. Found. Comput. Sci. 15 (2004) 317-327.

[16] A. Thue, Über unendliche Zeichenreihen, Norske vid. Selsk. Skr. Mat. Nat. Kl. 7 (1906), 1-22. Reprinted in Selected Mathematical Papers of Axel Thue, edited by T. Nagell, Universitetsforlaget, Oslo (1977) 139-158.

[17] A. Thue, Über die gegenseitige Lage gleicher Teile gewisser Zeichenreihen, Norske vid. Selsk. Skr. Mat. Nat. Kl. 1 (1912) 1-67. Reprinted in Selected Mathematical Papers of Axel Thue, edited by T. Nagell, Universitetsforlaget, Oslo (1977) 413-478.

[18] A.I. Zimin, Blocking sets of terms. Math. USSR Sbornik 47 (1984) 353-364. English translation. 